\title{
Análise temporal e espacial do número de casos e incidência de diarreia no Brasil no período de 2003 a 2016
}

O conhecimento acerca dos dados epidemiológicos do registro e incidência de diarreia no país é fundamental o dimensionamento das operações e processos envolvidos na cadeia de gerenciamento de saúde pública no âmbito nacional. Deste modo, o presente trabalho objetivou levantar o número de casos registrados de diarreia nos estados brasileiros, e calcular a incidência desta doença, no período de 2003 a 2016. Para isto, os dados necessários para esta pesquisa foram coletados em base de dados apropriadas e submetidas à análise temporal e espacial. Os resultados apontam que o perfil de diarreia no Brasil passou por transformações ao longo do período analisado, sendo que em 2003 poucos estados apresentavam registro desta doença, entretanto, com o passar dos anos todos os estados brasileiros apresentavam registro de casos, com destaque para os estados da região norte e nordeste, além dos estados de Minas Gerais, Paraná, Rio de Janeiro São Paulo e Santa Catarina que nos últimos anos apresentarem números elevados. Em relação à incidência foi apontado que o estado Amazonas era o estado que apresentava maior incidência de diarreia nos anos de 2003 (78\%) e 2004 (41\%), entretanto, a prevalência desta doença nesta região foi reduzindo e nos últimos anos analisados (2009 a 2016) a taxa de incidência de diarreia se tornou mais equilibrada entre os estados brasileiros. Sendo que o maior índice desta afecção nos últimos anos foi encontrado no estado de Santa Catarina, com incidência em torno de 4 a $12 \%$ do total de registros no país.

\section{Temporal and spatial analysis of the number of cases and incidence of diarrhea in Brazil from 2003 to 2016}

The knowledge about the epidemiological data of the registry and incidence of diarrhea in the country is fundamental the dimensioning of the operations and processes involved in the chain of public health management at the national level. Therefore, the present study aimed to estimate the number of cases of diarrhea in the Brazilian states, and to calculate the incidence of this disease, from 2003 to 2016 . For this, the necessary data for this research were collected in appropriate database and submitted to temporal and spatial analysis. The results indicate that the profile of diarrhea in Brazil underwent transformations throughout the analyzed period, and in 2003 few states presented a record of this disease, however, over the years all Brazilian states presented case records, with emphasis on the states of the north and northeast, as well as the states of Minas Gerais, Paraná, Rio de Janeiro, São Paulo and Santa Catarina, which in recent years have presented high numbers. Regarding the incidence, it was pointed out that the state of Amazonas was the state with the highest incidence of diarrhea in 2003 (78\%) and 2004 (41\%); however, the prevalence of this disease in this region was reduced and in the last years analyzed (2009 to 2016) the incidence rate of diarrhea became more balanced among the Brazilian states. The highest index of this condition in recent years was found in the state of Santa Catarina, with an incidence of around 4 to $12 \%$ of the total number of records in the country.

Keywords: Incidence of diarrhea; Temporal analysis.

Topic: Epidemiologia

Reviewed anonymously in the process of blind peer.

Claudiomir da silva dos santos (ii

Instituto Federal do Sul de Minas, Brasil

http://lattes.cnpq.br/7460335760795185

http://orcid.org/0000-0002-0007-7273

claudiomirsilvasantos@gmail.com

Fabrício dos Santos Rita (iD

Instituto Federal do Sul de Minas, Brasil

http://lattes.cnpq.br/9009240714607346

http://orcid.org/0000-0003-2009-3673

fabriciosantosrita@gmail.com

Monise Martins da Silva

Universidade do Estado de Minas Gerais, Brasil

http://lattes.cnpq.br/6516503063243721

http://orcid.org/0000-0001-9141-4775

monisemsilva@gmail.com

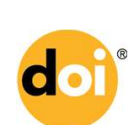

DOI: 10.6008/CBPC2236-9600.2021.002.0018
Received: $17 / 04 / 2021$

Approved: 12/05/2021

\author{
Ronei Aparecido Barbosa (D) \\ Instituto Federal do Sul de Minas, Brasil \\ http://lattes.cnpq.br/4964015135722519 \\ http://orcid.org/0000-0002-4850-7244
}

ronei.barbosa@muz.ifsuldeminas.edu.br

\section{Referencing this:}

SANTOS, C. S.; RITA, F. S.; SILVA, M. M.; BARBOSA, R. A.. Análise temporal e espacial do número de casos e incidência de diarreia no Brasil no período de 2003 a 2016. Scire Salutis, v.11, n.2, p. 157-162, 2021. DOI: http://doi.org/10.6008/CBPC2236-9600.2021.002.0018 



\section{INTRODUÇÃO}

Embora adquirida como um direito, na prática, os serviços de saneamento básico deixam de englobar uma parcela significativa da população mundial. Segundo Araújo et al. (2013) cerca de 2,4 bilhões de pessoas no mundo não têm acesso a qualquer tipo de saneamento básico e aproximadamente 1,1 bilhão de pessoas não têm acesso à água de boa qualidade. No Brasil, o saneamento básico é garantido pela Constituição e estabelecido pela Lei $11.445 / 2007$, a qual define normas nacionais que devem ser executadas como um conjunto de serviços que conferem proteção e qualidade de vida à população (BRASIL, 2007).

Apesar de ser um direito a todo cidadão, a construção deste cenário ideal na realidade das regiões mais pobres esbarra nos problemas sociais tão conhecidos nos países em desenvolvimento. A urbanização descontrolada, a desigualdade social e as condições precárias de acesso a serviços públicos são alguns dos inúmeros entraves que a promoção da saúde precisa superar. Ao viverem em condições sanitárias não adequadas, principalmente no que se refere ao contato com esgoto e água não tratada, grande parte da população está exposta diretamente a uma série de doenças de veiculação hídrica (OLIVEIRA et al., 2017). O uso da água imprópria para consumo impede a higienização do ambiente, não oferece segurança para as refeições e promove condições para a ocorrência do ciclo de doenças transmitidas por parasitos, vírus e bactérias e que associado ás características ambientais e do clima tendem a agravar ainda mais os surtos destas doenças (RUFINO et al., 2015).

As doenças infecciosas (grande parte associadas à veiculação hídrica) matam duas vezes mais que o câncer, sendo que as diretamente associadas à água levam a óbito quase o mesmo número que as doenças de pessoas que fumam (ARAÚjO et al., 2013).

Dentre as principais doenças de veiculação hídrica, as diarreicas agudas (DDA) constituem uma importante preocupação para a saúde pública, que quando somadas são responsáveis por cerca de 1,8 milhão de mortes todo ano no mundo, sendo que o maior número de mortes ocorre nos países em desenvolvimento. De todas as mortes ocorridas em decorrência das DDA, 90\% foram de crianças menores de 5 anos (ARAÚJO et al., 2013).

De fato, as doenças diarreicas são uma das causas mais comuns de desequilíbrio da saúde das crianças. As ocorrências das DDA nesta faixa etária estão condicionadas a diversos fatores, entre eles: ao grau de exposição aos patógenos entéricos e pela suscetibilidade do organismo infantil, associada às condicionantes relacionados ao acesso a água tratada, saneamento básico, nutrição, temperatura e pluviosidade (MENEGUESSI et al., 2015). No Brasil, a diarreia constitui uma das principais doenças de veiculação hídrica e aproximadamente 33 milhões de casos de diarreia foram notificados entre 2000 e 2011, sendo a maioria dos acometidos crianças menores de 1 ano (BRASIL, 2012).

Sendo, portanto, diarreia um assunto de grande preocupação e importância para a saúde humana, o conhecimento acerca da prevalência desta doença no país é de interesse público, e pode auxiliar na tomada de decisões de estratégias de controle e prevenção em áreas consideradas prioritárias para 
atuação. Neste sentido, o objetivo deste trabalho foi traçar um perfil epidemiológico, temporal e espacial da diarreia, em todos os estados brasileiros, durante o período de 2003 a 2016.

\section{METODOLOGIA}

Realizou-se um estudo com abordagem quantitativa, retrospectivo, descritivo e epidemiológico, com distribuição temporal e espacial, a partir de dados secundários relacionados à diarreia, considerado todo o território nacional, seus 26 estados e o Distrito Federal. Os dados relacionados à diarreia do período de janeiro de 2003 a janeiro de 2016 foram obtidos por meio do Sistema Informatizado de Vigilância Epidemiológica de Doenças Diarreicas Agudas- SIVEPE DDA por meio da base de dados http://www.saude.gov.br/sivep_dda referentes às notificações dos casos de diarreias por estado, sendo excluídos da análise os casos não residentes no Brasil e casos com duplicidades de notificação.

Os dados referentes à população foram obtidos no sítio eletrônico do Instituto Brasileiro de Geografia e Estatística. O coeficiente de incidência de diarreia foi calculado utilizando-se o número de casos registrados da doença por 100.00 habitantes, dividido pelo número de habitantes. Com base nas informações geradas foram criados mapas temáticos do estudo. . Através dos Softwares Microsoft Word e Excel $365^{\circledR}$, pertencente ao pacote Microsoft Office $365^{\circledR}$, Windows ${ }^{\circledR}$. Os dados levantados foram organizados e expostos por meio de tabelas explicativas.

\section{RESULTADOS E DISCUSSÃO}

A diarreia é responsável mundialmente por cerca de 525.000 mortes de crianças por ano e é a segunda causa de morte em menores de 5 anos de idade (OMS, 2017). Esta doença acomete principalmente a população de países de baixa renda, muitas vezes submetidas a precárias condições de saneamento básico.

No que diz respeito ao Brasil, a mortalidade em decorrência desta doença diminuiu no período de 1990 a 2010 (MARINHO et al., 2016) seguindo a tendência mundial, conforme descrito por Walker et al. (2012). Neste estudo os autores apresentaram estimativas que apontaram o decréscimo na incidência de diarreia em países de baixa e média renda, de 3,4 registros infantil por ano em 1990 para 2,9 registros infantil por ano em 2010. Em decorrência deste decréscimo, nos 139 países avaliados observou-se que de 1990 para 2010 os registros de diarreia infantil foram de 1,9 bilhão em 1990 para 1,7 bilhão em 2010, um número ainda bastante expressivo.

Embora o trabalho de Marinho et al. (2016) indique que a mortalidade em decorrência de diarreia tenha diminuído no Brasil, esta doença representa ainda hoje um enorme desafio para o sistema básico de saúde brasileiro, principalmente nas comunidades pertencentes às regiões mais pobres. Os resultados obtidos neste estudo demonstram que entre o período de 2003 a 2016, foram registrados no Brasil, um total de 39.996.295 casos de diarreia.

No período de 2003 a 2016 é possível observar que o perfil de diarreia no Brasil passou por 
transformações ao longo do período analisado. Em 2003 os estados com maior número de casos eram o Amazonas, com 87.068 registros, seguido por Mato Grosso do Sul (5.642 registros), Rio Grande do Norte (3.033 registros), Espírito Santo (889 registros), São Paulo (243 registros) e Ceará (11 registros) enquanto que nos demais estados não há registros de nenhum caso. Em 2004, Amazonas (73.770 registros) permanece com o maior número de registros de casos de diarreia, seguido por Amapá (13.357 registros), Mato Grosso do Sul (5.438 registros), Rio Grande do Norte (3.391 registros), Espírito Santo (3.105 registros), Santa Catarina (2.272 registros), Pará (833 registros) e Pernambuco (189 registros), também sem nenhum registro em outros estados brasileiros. No ano de 2005 o estado do Amazonas manteve-se em primeiro lugar (95.012 registros), seguido por Paraíba (45.132 registros), Amapá (23.155 registros), Sergipe (5.254 registros), Mato Grosso do Sul (5.134 registros), Minas Gerais (336 registros), Distrito Federal (5 registros) e Tocantins (2 registros). A partir de 2006, a maior parte dos estados já citados aparecem com registros ainda maiores de casos de diarreia tais como Amazonas, Paraíba, Espirito Santo, Rio Grande do Norte, Paraíba, Mato Grosso do Sul, Santa Catarina e Sergipe e em estados antes não citados tais como Alagoas, Acre, Bahia, Distrito Federal, Mato Grosso, Pará, Paraná, Rio Grande do Sul, Rondônia e Roraima os casos de diarreias de um ano para o outro aumentaram de forma expressiva, com destaque para os estados do Ceará, Rio de Janeiro e Maranhão que passaram de 0 registros em 2005 para 189.675, 188.890 e 131.334 registros em 2006, respectivamente. Os únicos estados no ano de 2006 em que não foi registrado nenhum caso de diarreia foram Goiás, Minas Gerais, Piauí e Tocantins.

Em 2007, o único estado brasileiro que não apresenta registro de casos de diarreia é o de Minas Gerais, a ocorrência maior continua sendo em estados do Norte e Nordeste, tais como Amazonas (158.057 registros), Bahia (233.940 registros), Ceará (230.696 registros), Pará (168.441 registros), Pernambuco (217.770 registros). São Paulo também apresenta número de expressivos de casos registrados (383.526 registros), com elevado crescimento para os próximos anos analisados, sendo registrado neste estado nos anos de 2008 a 2015 mais de um milhão de casos por ano, provavelmente pela alta concentração populacional.

Vaz et al. (2017) apontam uma correlação positiva entre o número de internações no estado de São Paulo e o nível de escolaridade materna (1 ciclo fundamental incompleto) no período de 2008 a 2012, mostrando assim que fatores tais como higiene, imunização e amamentação podem influenciar diretamente no surgimento de gastroenterites, e nos registros de casos desta doença. Estes autores descrevem ainda que foram notificadas no estado 34.802 internações por diarreia em crianças menores que 4 anos, no mesmo período. Um estudo realizado por Martins et al. (2016) apontou após análise temporal que aconteceram no estado de São Paulo no período de 2000 a 2012, 2886 óbitos de crianças menores de 5 anos de idade em decorrência de doenças infecciosas intestinais, e que a taxa de mortalidade neste mesmo estado, para esta faixa etária, diminuiu em 10,5\% ao ano.

Para os demais estados do Brasil, o presente estudo mostra que para os anos de 2008 a 2016, o registro de diarreia acontece praticamente em todos estados brasileiro, com destaque para os estados do 
Nordeste, e Norte e em alguns anos registros expressivos em outros estados (Paraná, Minas Gerais, entre outros). Um estudo realizado no período de 2000 a 2010, para idades de 0 a 1 ano e de 1 a 4 anos apontou que houve uma tendência de declínio da mortalidade por diarreia no Brasil nestas faixas etárias avaliadas. Já para as internações o estudo demonstrou que houve discreto declínio para os menores de 1 ano, enquanto para a faixa etária de 1 a 4 anos não se mostrou significante.

A região Norte e Nordeste foram as que apresentaram piores índices de mortalidade e maior porcentagem de hospitalizações (MENDES et al., 2013), o que corrobora com os dados também obtidos no presente trabalho, muito embora o conjunto de dados sejam bem diferentes, ambos as pesquisas apontam que a região Norte e Nordeste representa umas das regiões com maior número de casos desta doença.

A fim de retratar resultados mais explicativos do perfil epidemiológico da diarreia, o número de casos desta doença por estado foi utilizado para o cálculo da sua incidência, descrevendo melhor as áreas que necessitam de maior atenção, ou seja, que tenham apresentado maior incidência desta doença.

Tabela 1: Incidência anual dos casos de diarreia por estado Brasileiro no período de 2003 a 2016.

\begin{tabular}{|c|c|c|c|c|c|c|c|c|c|c|c|c|c|c|}
\hline ESTADOS & 2003 & 2004 & 2005 & 2006 & 2007 & 2008 & 2009 & 2010 & 2011 & 2012 & 2013 & 2014 & 2015 & 2016 \\
\hline ACRE & 0 & 0 & 0 & 1,39 & 4,86 & 4,86 & 3 & 3,71 & 4,26 & 4,63 & 3,73 & 4,4 & 4,14 & 3,5 \\
\hline ALAGOAS & 0 & 0 & 0 & 0,03 & 1,08 & 1,94 & 1,05 & 2,1 & 2,11 & 2,39 & 3,75 & 2,68 & 2,73 & 2,67 \\
\hline AMAPÁ & 0 & 33,15 & 27,51 & 3,9 & 2,4 & 2,54 & 2,44 & 1,83 & 2,07 & 2,06 & 1,96 & 2,39 & 2,42 & 2,64 \\
\hline AMAZONAS & 77,51 & 40,45 & 24,94 & 5,31 & 3,81 & 3,18 & 3,42 & 3,32 & 3,01 & 3,21 & 2,62 & 2,9 & 3,18 & 2,99 \\
\hline BAHIA & 0 & 0 & 0 & 0 & 3,8 & 4 & 3,89 & 3,44 & 3,07 & 2,95 & 3,08 & 2,33 & 2,06 & 2,04 \\
\hline CEARÁ & 0,01 & 0 & 0 & 5,72 & 4,09 & 3,54 & 3,53 & 2,8 & 3,88 & 2,59 & 3,5 & 2,72 & 2,99 & 3,93 \\
\hline DISTRITO FEDERAL & 0 & 0 & 0 & 0,16 & 1,23 & 1,33 & 1,09 & 1,21 & 0,94 & 0,86 & 0,71 & 0,9 & 0,43 & 0,36 \\
\hline ESPÍRITO SANTO & 4,35 & 9,36 & 6,16 & 13,91 & 6,41 & 6,52 & 8,62 & 8,18 & 8,04 & 7,74 & 6,19 & 7,14 & 6,68 & 6,92 \\
\hline GOIAS & 0 & 0 & 0 & 0 & 0,19 & 1,98 & 1,7 & 2,29 & 3,57 & 3,86 & 3,29 & 3,76 & 3,65 & 3,7 \\
\hline MARANHÃO & 0 & 0 & 0 & 9,57 & 4,72 & 4,02 & 4,49 & 4,28 & 4,06 & 3,23 & 3,56 & 3,62 & 3,3 & 3,47 \\
\hline MATO GROSSO & 0 & 0 & 0 & 11,66 & 6,4 & 5,4 & 5,35 & 5,36 & 4,36 & 4,09 & 3,68 & 3,74 & 3,71 & 3,28 \\
\hline MATO GROSSO DO SUL & 11,5 & 6,83 & 3,09 & 4,44 & 4,31 & 4,38 & 4,43 & 5,02 & 4,42 & 4,34 & 3,68 & 3,67 & 3,29 & 2,6 \\
\hline MINAS GERAIS & 0 & 0 & 0,07 & 0 & 0 & 0 & 0 & 0,01 & 0,01 & 4,48 & 4,15 & 5,45 & 5,26 & 4,52 \\
\hline PARA & 0 & 0,59 & 0 & 1,7 & 5,25 & 4,61 & 4,79 & 3,98 & 3,68 & 3,92 & 3,76 & 3,91 & 3,78 & 3,94 \\
\hline PARAIBA & 0 & 0 & 29,51 & 12,05 & 6,01 & 5,34 & 5 & 3,88 & 3,74 & 3,1 & 5,44 & 3,84 & 4,75 & 5,03 \\
\hline PARANA & 0 & 0 & 0 & 0,1 & 0,52 & 0,54 & 0,6 & 3,26 & 4,99 & 4,63 & 3,96 & 5,62 & 5,43 & 5,18 \\
\hline PERNAMBUCO & 0 & 0,12 & 0,07 & 0,43 & 6,15 & 5,58 & 6,15 & 4,74 & 4,37 & 4,07 & 5,94 & 4,38 & 4,61 & 5,08 \\
\hline PIAUI & 0 & 0 & 0 & 0 & 3,9 & 4,39 & 4,02 & 3,35 & 3,59 & 3,04 & 3,69 & 3,73 & 3,25 & 4,07 \\
\hline RIO DE JANEIRO & 0 & 0 & 0 & 2,21 & 1,1 & 0,83 & 1,08 & 0,88 & 0,74 & 0,61 & 0,65 & 0,69 & 0,68 & 0,47 \\
\hline RIO GRANDE DO NORTE & 6,59 & 4,17 & 1,99 & 9,95 & 4,52 & 4,03 & 4,43 & 4,27 & 4,55 & 3,95 & 5,73 & 3,93 & 5,05 & 4,75 \\
\hline RIO GRANDE DO SUL & 0 & 0 & 0 & 0,64 & 3,66 & 4,04 & 3,53 & 3,99 & 3,38 & 3,59 & 2,73 & 2,9 & 2,75 & 2,77 \\
\hline RONDÔNIA & 0 & 0 & 0 & 4,89 & 3,32 & 3,29 & 4,09 & 3,61 & 3,33 & 2,95 & 2,72 & 2,99 & 3,07 & 1,95 \\
\hline RORAIMA & 0 & 0 & 0 & 5,58 & 3,46 & 2,49 & 2,88 & 2,59 & 2,68 & 2,5 & 2,33 & 2,4 & 2,49 & 2,7 \\
\hline SANTA CATARINA & 0 & 5,33 & 2,32 & 3,94 & 9,39 & 11,23 & 9,53 & 12 & 7 & 4 & 9 & 11 & 9 & 11 \\
\hline SÃO PAULO & 0,03 & 0 & 0 & 0 & 1,48 & 2,23 & 2,38 & 2,79 & 2,91 & 3,14 & 2,8 & 3,12 & 3 & 3,01 \\
\hline SERGIPE & 0 & 0 & 4,35 & 2,42 & 1,06 & 1,1 & 1,09 & 0,88 & 1,14 & 1,54 & 1,12 & 0,69 & 0,87 & 0,73 \\
\hline TOCANTINS & 0 & 0 & 0 & 0 & 6,82 & 7,08 & 6,54 & 7,96 & 6,43 & 5,16 & 5,73 & 6,35 & 6,05 & 6,81 \\
\hline
\end{tabular}

\section{CONCLUSÕES}

O conjunto de dados analisados permitiu apontar que o número de pessoas acometidas pela diarreia no país ainda é alto na maior parte dos estados brasileiros. Amazonas era o estado que apresentava maior incidência de diarreia nos anos de 2003 e 2004, entretanto, a prevalência desta doença nesta região foi reduzindo, e ao longo dos anos seguintes observou-se um aumento na incidência de diarreia nos demais estados brasileiros, com destaque para Santa Catarina que desde 2007 é o estado que 
apresenta maior incidência desta doença.

\section{REFERÊNCIAS}

ARAÚJO, M. F. F.. Doenças de veiculação hídrica: conhecendo e prevenindo. Natal: UFRN, 2013.

BRASIL. Lei no 11445. Estabelece diretrizes nacionais para o saneamento básico. Brasília: DOU 2007.

BRASIL. Análise da situação das doenças transmissíveis no Brasil no período de 2000 a 2010. Brasília: Ministério da Saúde, 2012.

BUSATO, M. A.; LUTINSKI, J. A.; SOUZA, M. F.; CECHIN, F.; FILHO, D. G.; PERIPOLLI, C.; MARANGONI, S. R.; CONSTANCI, C.. Distribuição de doenças diarreicas agudas em Municípios do estado de Santa Catarina. Hygeia, v.9, n.16, p.18, 2013.

IMADA, K. S.; ARAÚJO, T. S.; MUNIZ, P. T.; PÁDUA, V. T.. Socioeconomic, hygienic, and sanitation factors in reducing diarrhea in the Amazon. Revista de Saúde Pública, v.50, p.77, 2016. DOI: http://dx.doi.org/10.1590/s1518$\underline{8787.2016050006505}$

MARINHO, F.; PASSOS, V. M. A.; FRANÇA, E. B.. New century, new challenges: changes in the burden of disease profile in Brazil, 1990-2010. Epidemiologia e Serviços de Saúde, v.25, n.4, p.713-724, 2016. DOI: http://dx.doi.org/10.5123/s1679$\underline{49742016000400005}$

MARTINS, R. S.; EDUARDO, M. B. P.; NASCIMENTO, A. F.. Time trends in mortality from intestinal infectious diseases among children under five years old, in São Paulo State, Brazil, 2000-2012. Epidemiologia e Serviços de Saúde, v.25, n.3, p.541-552, 2016. DOI: http://dx.doi.org/10.5123/s167949742016000300010.

MENDES, P. S. A.; RIBEIRO, H. C.; MENDES, C. M. C..

Temporal trends of overall mortality and hospital morbidity due to diarrheal disease in Brazilian children younger than 5 years from 2000 to 2010. Jornal de Pediatria, v.89, n.3, p.315-325, 2013. DOI: http://dx.doi.org/10.1016/i.jped.2012.10.002.

MENEGUESSI, G. M.; MOSRRI, R. M.; SEGATTO, T. C. V.; REIS, P. O.. Morbimortalidade por doenças diarreicas agudas em crianças menores de 10 anos no Distrito Federal, Brasil, 2003 a 2012. Epidemiologia e Serviços de Saúde, v.24, p.721-730, 2015. DOI: http://dx.doi.org/10.5123/S1679$\underline{49742015000400014}$

OLIVEIRA, J. S. C.; MEDEIRO, A. M.; CASTOR, L. G.; CARMOS, R. F.; BEVILACQUA, P. D.. Soluções individuais de abastecimento de água para consumo humano: questões para a vigilância em saúde ambiental. Cadernos Saúde Coletiva, v.25, n.2, 2017. DOI:

http://dx.doi.org/10.1590/1414-462×201700020371

ORGANIZAÇÃO MUNDIAL DA SAÚDE. Diarrhoeal disease. OMS, 2017.

RUFINO, R.. Diarrhea outbreaks in northeastern Brazil in 2013, according to media and health information systems Surveillance of climate risk and health emergencies. Ciência e Saúde Coletiva, v.3, p.777-788, 2016. DOI: http://dx.doi.org/10.1590/1413-81232015213.17002015

VAZ, F. P. C.; NASCIMENTO, L. C.. Spatial distribution for diarrhea hospitalization in São Paulo State. Revista Brasileira de Saúde Materno Infantil, v.17, n.3, p.475-482, 2017. DOI: http://dx.doi.org/10.1590/1806-93042017000300004

WALKER, C. L. F.. Diarrhea incidence in low- and middleincome countries in 1990 and 2010: a systematic review. BMC Public Health, v.12, n.1, p.1-7, 2012. DOI: http://dx.doi.org/10.1186/1471-2458-12-220

A CBPC - Companhia Brasileira de Produção Científica (CNPJ: 11.221.422/0001-03) detém os direitos materiais desta publicação. Os direitos referem-se à publicação do trabalho em qualquer parte do mundo, incluindo os direitos às renovações, expansões e disseminações da contribuição, bem como outros direitos subsidiários. Todos os trabalhos publicados eletronicamente poderão posteriormente ser publicados em coletâneas impressas sob coordenação da Sustenere Publishing, da Companhia Brasileira de Produção Científica e seus parceiros autorizados. Os (as) autores (as) preservam os direitos autorais, mas não têm permissão para a publicação da contribuição em outro meio, impresso ou digital, em português ou em tradução. 